\title{
INFORME FINAL SEMILLERO DE INVESTIGACIÓN FILOSOFÍA E INFANCIA 2020-2 FILOINFANCIA
}

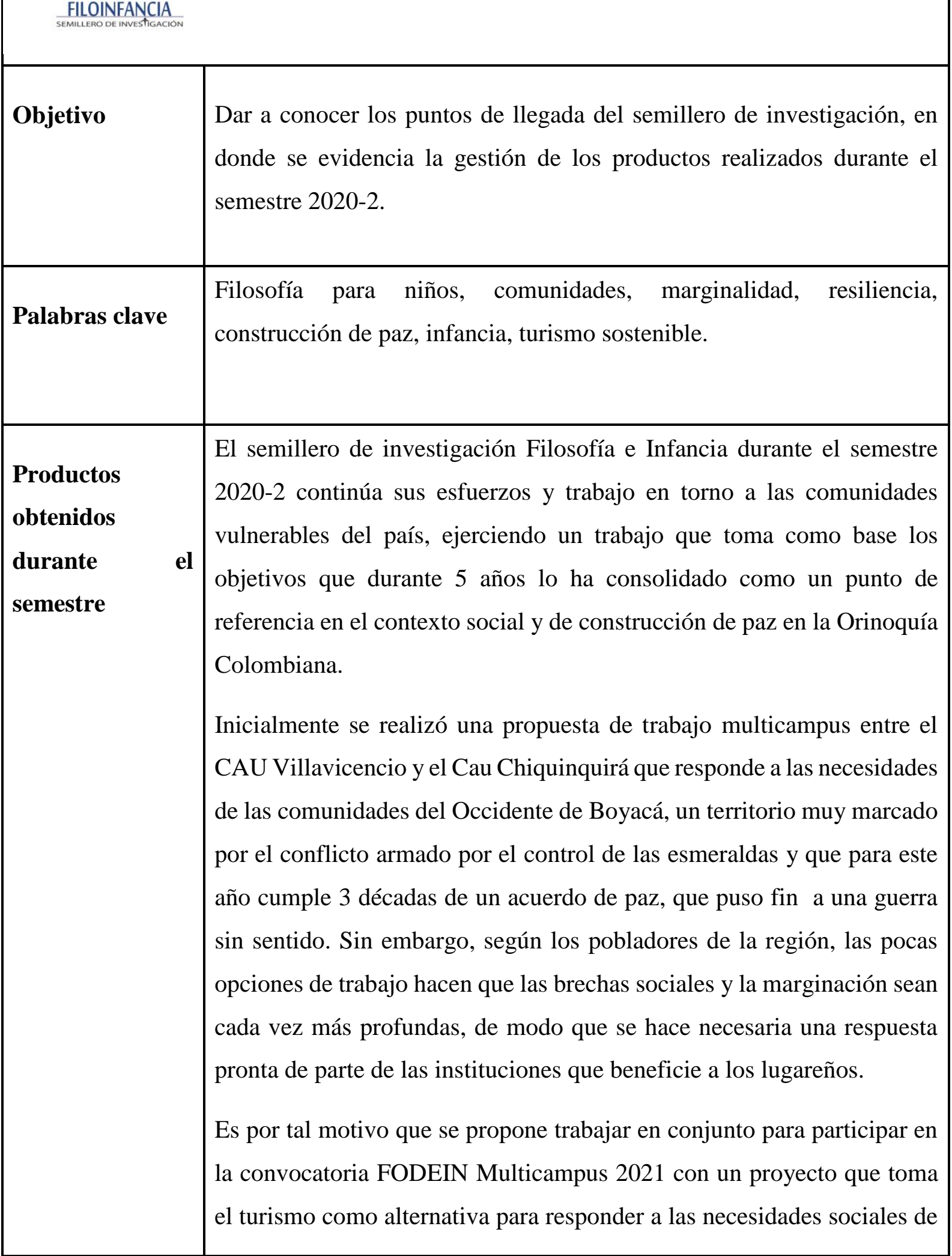


la región y tiene como objetivo Diseñar un plan convencional de desarrollo turístico para San Pablo de Borbur en Boyacá, bajo el enfoque de turismo sostenible en su primera fase de diagnóstico y avance.

Este proyecto se encuentra en fase de revisión por parte de pares externos y cuenta con el apoyo de instituciones como BOYAPAZ, CORESCOL, la alcaldía municipal de San Pablo de Borbur y la Finca San Luis de este mismo municipio.

Paralelo a este proceso, el semillero tiene la oportunidad de publicar un libro que hará parte de la edición especial Escala 440 de la Universidad Santo Tomás. Esto se presenta como una oportunidad ideal para dar a conocer el trasegar del semillero durante los últimos años en la región de la Orinoquía y los resultados obtenidos en este tiempo.

En dicho libro, denominado memorias del conflicto - de infancia víctima a resiliente en el departamento del Meta, se condensan las experiencias investigativas del semillero, los eventos, ponencias realizadas a nivel nacional e internacional, las experiencias investigativas en las convocatorias FODEIN en las que ha participado el semillero, un artículo que realza la importancia del arte como expresión de la realidad social de nuestros pueblos y un capítulo en el cual se publica una entrevista realizada a Walter Kohan, uno de los principales exponentes de la filosofía para niños en el mundo.

Otro de los productos que se gestionaron durante el segundo semestre del año 2020 es un artículo para la revista Sol de Aquino que se titula grupo de investigación NAKOTA: una trayectoria investigativa desde el conflicto armado hasta el emprendimiento; (es pertinente aclarar en este momento de que el semillero Filosofía e Infancia hace parte del Grupo de Investigación NAKOTA, de modo que hay una participación directa en las publicaciones del grupo por parte de integrantes del semillero).

Como parte de la divulgación de los procesos investigativos y los hallazgos realizados por el semillero se realizó la postulación de una 
ponencia en la IV bienal latinoamericana y caribeña en primera infancia, niñez y juventud que se realizará el próximo año entre mayo y junio en la ciudad de Manizales, Caldas, Colombia, un escenario que será propicio para seguir hablando de paz, infancia y resiliencia.

Como parte del proceso emprendido por el semillero de investigación en alianza con el CAU chiquinquirá, en aras de dar a conocer el contexto del Occidente de Boyacá y como parte de la preparación de los investigadores del semillero en vista de la oportunidad de realizar un proceso investigativo en dicha región, en el mes de septiembre del presente año se realizó el evento contexto histórico del Occidente de Boyacá, en el que se contó con la participación del pbro. Victor Julio Mendieta Vergel, sacerdote de la diócesis de Chiquinquirá e historiador de la Academia de Historia Boyacense.

En esta oportunidad el sacerdote hizo referencia a la cultura Muzo Colima, que es una cultura ancestral que habitó el Occidente de Boyacá, el sur de Santander y el norte de Cundinamarca en la época prehispánica y que se mestizó con los colonos españoles hasta desaparecer del contexto territorial. Dicho aporte contribuyó a la formación y reconocimiento de la realidad de la sociedad boyacense en esta provincia.

Uno de los proyectos del semillero de investigación para este año es la necesidad de hacer parte de la Red Colombiana de Filosofía para Niños y por lo tanto está trabajando en el cumplimiento de los requisitos necesarios para pertenecer a dicha red, entre ellos, un video en el que se difunda los logros del semillero, por lo cual, los integrantes del mismo se han dado a la tarea de organizar un producto audiovisual muy completo y que además sintetice los alcances y logros realizados en los últimos años. Esta gestión es realizada por cada uno de los miembros del semillero filoinfancia.

Otro de los aspectos que se han abordado en el proceso investigativo del semillero filoinfancia es un capítulo de libro en el que se evidencia el estado de investigación en el la sede de la universidad Santo Tomás en 
Villavicencio, el cual saldrá publicado en la primera semana del mes de diciembre del presente año bajo el título la investigación en la USTA, sede Villavicencio: capacidades CTel desde los grupos de investigación; el capítulo se encuentra bajo el nombre Grupo de Investigación Nakota: sociedad, emprendimiento, innovación e industria. Aborda todos los temas concernientes a los procesos investigativos desde los distintos semilleros de investigación que lo integran, entre ellos el semillero filoinfancia.

Como parte de la investigación emprendida por el semillero SIEET del grupo de Investigación Nakota en la convocatoria FODEIN 2020, se participó en el proceso de construcción de los signos distintivos del semillero filoinfancia y del grupo de investigación, un proceso que permitió a los integrantes del semillero ir a las mismas raíces y plasmar en los signos el ser y sentir de quienes lo integran.

la influencia de los procesos desarrollados por el semillero de investigación durante los últimos 5 años han sido de gran relevancia en el ámbito educativo y de formación en los colegios de Villavicencio en filosofía para niños. En este proceso ha logrado articular actividades con el sistema MARFIL de la Universidad Minuto de Dios, el cual hizo una invitación a integrantes del semillero de investigación a participar en un evento internacional con maestros de escuelas y colegios de México, quienes buscan implementar la filosofía para niños en escuelas de ese país.

Este espacio permitió a los participantes arrojar luces sobre los distintos aspectos de la filosofía para niños en las aulas, de las repercusiones de la implementación de esta cátedra en la aulas y de su trascendencia en el ámbito social, en el contexto de las instituciones educativas y en las mismas familias de los estudiantes, generando además en ellos actitudes críticas y propositivas frente a las problemáticas de la sociedad.

Finalmente se resaltar la participación del semillero de investigación en el programa el Puente en Casa de la Universidad Minuto de Dios el 


\begin{tabular}{|c|c|}
\hline & $\begin{array}{l}\text { pasado } 12 \text { de noviembre, un espacio en el cual se tuvo la oportunidad } \\
\text { también de dar a conocer la génesis de la investigación del semillero en } \\
\text { torno a los menores víctimas del conflicto armado colombiano y las } \\
\text { acciones que desde ese entonces ha emprendido en favor de aquellos que } \\
\text { no son escuchados. }\end{array}$ \\
\hline $\begin{array}{l}\text { Referentes } \\
\text { bibliográficos }\end{array}$ & 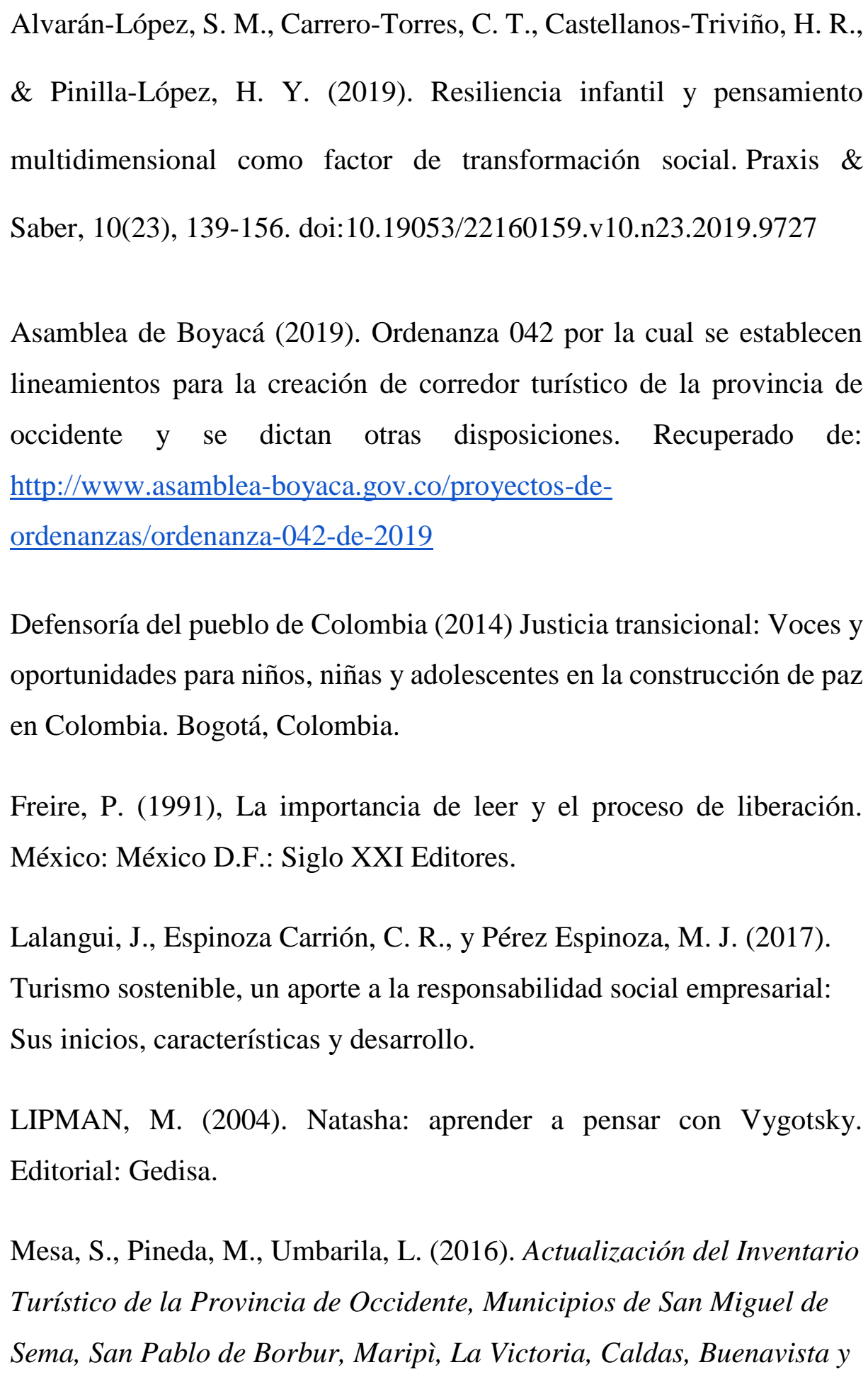 \\
\hline
\end{tabular}


Zona de Manejo Especial (zme) Puerto Boyacá [Trabajo de grado].

UPTC. Recuperado de:

https://repositorio.uptc.edu.co/bitstream/001/2715/1/TGT_1336.pdf

Nieto, Patricia (2010). Relatos autobiográficos de víctimas del conflicto armado: una propuesta teórico-metodológica. Revista de Estudios Sociales, número 3.

OIM (2014). Impacto del conflicto armado en el estado psicosocial de niños, niñas y adolescentes. Informe conjunto con ICBF y UNICEF. Bogotá, Colombia: ICBF

Pérez, M. G. (2016). Pensamiento Multidimensional y Práctica Filosófica Según Mathew Lipman. Parecidos de familia, Propuestas actuales de filosofía para niños.

SÁTIRO, A. (2012) “«Pedagogía para una ciudadanía creativa»” Tesis doctoral "“. Doctorado en la Facultad de Pedagogía de la Universidad de Barcelona. Copyright depósito legal: B. 33212-2012. Publicación: Tesis doctorales en Xarxa: www.tdx.cat/handle/10803/96169

Unidad de víctimas. (18 de 04 de 2020). Registro único de víctimas. Obtenido de Registro único de víctimas: https://www.unidadvictimas.gov.co/es/registro-unico-de-victimas$\mathrm{ruv} / 37394$

Links asociados a la gestión del semestre:
1) Vídeo semillero filoinfancia.

https://drive.google.com/file/d/1bMtVjA2yYI8Bfrdvq8Niotu3jTzqCes/view

2) Uniminuto radio: El puente en casa / comunidades de diálogo y conflicto armado 
https://www.youtube.com/watch?v=lWupjYP-7-

w\&ab_channel=MARFIL\%3AEducaci\%C3\%B3nFilos\%C3\%B3ficayC reatividadSocial

3) constancia de libro de investigación postulado Escala 440.

https://drive.google.com/file/d/1gIFfkNJbbNBm1YGrb3pdz3M_nisCiv $\underline{1 F / v i e w ? u s p=s h a r i n g}$

4) Constancia de artículo para revista Sol de Aquino.

https://drive.google.com/file/d/19yIn8JTjYdQJ5pb2RXHKQYZrEVclr oSU/view? usp=sharing

5) Evidencia de propuesta para Fodein 2021 en proceso de evaluación.

https://drive.google.com/file/d/1vsMVeKbG3U5mHvXNoNZjFTWfFq eNbb-F/view?

6) Capítulo de libro

https://drive.google.com/file/d/1Z75Li3T0LHcfdmRFfLv_yjzmBefYZ PjE/view? usp $=$ sharing

7) Signos distintivos

Logos semillero y grupo de investigación.

https://drive.google.com/file/d/1Ku2pbkkK7GdF_Yv2AQKpauRZlZts2 $\underline{\text { ClV/view? } \mathrm{usp}=\text { sharing }}$

https://drive.google.com/file/d/1YhAA6uARbPx071vvkzxpUBhekCka $\underline{\mathrm{dkPB} / \text { view? } \mathrm{usp}=\text { sharing }}$

Certificado de participación en el proceso. 
https://drive.google.com/file/d/1wtrKLgYAuU41c3Vg8jDsLVF-

NRhG9SHj/view?usp=sharing

8) Conversatorio con maestros de México

https://drive.google.com/open?id=1OWpvZYVH_8QGuHPdZLE_Pr4

MuNka0bU5

9) Dialogando sobre comunidades de diálogo y conflicto armado.

https://drive.google.com/file/d/1tR0JUt2pC3uD_1vPE6Dx8c8ievkJTR

YI/view?usp=sharing

10) Actas de reuniones de semillero

https://drive.google.com/drive/folders/12mLQmiVLu4T6zXPxRN7vKg jUfysTffas?usp=sharing

HÉCTOR RAFAEL CASTELLANOS TRIVIÑO

Docente asesor - semillero Filoinfancia

HENRY FERRER FLORIÁN DELGADILLO

Estudiante líder - semillero Filoinfancia
DAHANA VALENTINA NEIRA HENAO

Secretaria - semillero Filoinfancia 